\title{
LA SAGA DE LOS INGENIEROS BRITÁNICOS MANBY Y SU CONTRIBUCIÓN A LA INDUSTRIA DEL GAS EN FRANCIA Y ESPAÑA (1776-1884)
}

\author{
Mercedes Fernández-Paradas \\ Universidad de Málaga \\ Email: paradas@uma.es \\ ORCID iD: https://orcid.org/0000-0002-3049-0907 \\ Antonio Jesús Pinto Tortosa \\ Universidad Europea \\ Email: antoniojesus.pinto@universidadeuropea.es \\ ORCID iD: https://orcid.org/0000-0002-9921-568X
}

Recibido: 25 abril 2021; Aceptado: 15 mayo 2021

Cómo citar este artículo/Citation: Fernández-Paradas, Mercedes; Pinto Tortosa, Antonio Jesús (2021) "La saga de los ingenieros británicos Manby y su contribución a la industria del gas en Francia y España (1776-1884)", Asclepio, 73 (2): p561. https://doi.org/10.3989/ asclepio.2021/19

RESUMEN: El inglés Aaron Manby (1776-1850) es el iniciador de una saga, continuada por sus hijos, también ingenieros: Charles (18041884), John Richard (1813-1869), Joseph (1814-1862), y Edward (1816-1864). Todos ellos desempeñaron un papel muy relevante en la Europa del ochocientos, combinando su profesión como ingenieros con una intensa actividad industrial, que desarrollaron en Gran Bretaña, Francia y España. Aaron, el patriarca, fue el primero en construir un barco de hierro, que comunicó Gran Bretaña y Francia. En Francia levantó una gran industria metalúrgica a principios de la década de 1820, dedicándose a la construcción de este tipo de buques, y entre otros menesteres también abarcó la fabricación de equipamiento para el negocio gasista; de hecho, obtuvo la concesión para el alumbrado de París. A mediados de los años 1840 sus hijos Joseph y Edward marcharon a España, donde destacaron con un trabajo frenético en la industria del gas, así como en la desecación de lagunas, la construcción de ferrocarril, o la explotación minera. En este estudio se analiza el conjunto de sus actividades, subrayando su participación en el nacimiento de la industria española del gas, sin descuidar el resto de ámbitos en los que diversificaron sus inversiones.

PALABRAS CLAVE: Capital Extranjero; Despegue Económico; Industria del Gas; Ingenieros; Revolución Industrial.

\section{THE BRITISH MANBY ENGINEERS SAGA AND THEIR CONTRIBUTION TO THE GAS INDUSTRY IN FRANCE AND SPAIN} (1776-1884)

ABSTRACT: Aaron Manby (1776-1850) inaugurated a saga that his sons, civil engineers, too, continued: Charles (1804-1884), John Richard (1813-1869), Joseph (1814-1862), and Edward (1816-1864). They were relevant characters in 19 ${ }^{\text {th }}$ Century Europe, as they combined their work as engineers with an intense industrial activity, which they carried out in Great Britain, France, and Spain. The father, Aaron, was the first person to build a steel steamship in the World, in order to communicate Great Britain and France. In France he created a huge iron and steel industry in the 1820s, to build more ships using the same procedure, and he also invested in the gas industry, getting the concession to build the gas lighting of Paris. By mid-1840s his sons, Joseph and Edward, went to Spain, where they participated in the take-off of the gas industry, as well as the desiccation of lagoons, the building of the new railway lines, and the mining industry. In this article we focus on all their activities, highlighting their role in the birth of Spanish gas lighting, but we also describe the other activities in which they diversified their investments.

KEY WORDS: Economic Take-off; Engineers; Foreign Capital; Gas Industry; Industrial Revolution. 


\section{INTRODUCCIÓN}

La presente investigación constituye un análisis profundo de la familia Manby, estrechamente ligada al despertar de la industria europea en el tránsito del siglo XVIII al siglo XIX, con especial presencia en dos sectores: la siderurgia y el gas. El objetivo principal del artículo es estudiar el conjunto de actividades económicas de los integrantes de la saga, destacando las figuras de Aaron Manby, el padre, así como de sus hijos, sobre todo Charles y Edward Oliver. Los objetivos secundarios son: en primer lugar, destacar el peso de la transferencia de capital británico para el despegue industrial de las principales potencias de la Europa latina, centrando la atención inicialmente en Francia; en segundo lugar, subrayar la necesaria presencia del capital extranjero en el despegue industrial español, más tardío respecto al resto del continente, pero en el cual el capital británico y francés jugó un papel esencial, a través de apellidos tan destacados como los Partington, Lebon, o Manby, entre otros.

Para alcanzar tales objetivos, se ha procedido a realizar un exhaustivo estudio de fuentes documentales de diversa procedencia en perspectiva regional, contextualizando los avances identificados en el marco del desarrollo industrial europeo en la centuria decimonónica, con un enfoque metodológico propio de la historia económica. Destacan los fondos del Reino Unido: la documentación de la Staffordshire Record Office sobre las primeras actividades desarrolladas por el patriarca de la saga, Aaron Manby; los fondos de The National Archives, donde se ha localizado el testamento de Aaron Manby, así como la sucesión de pleitos a los que William Partington y Edward O. Manby debieron hacer frente por el desarrollo de la industria del gas en España; la documentación existente en la biblioteca de la London School of Economics (LSE), sobre el desarrollo del primer barco de hierro movido por vapor a cargo de Aaron Manby; los London Metropolitan Archives, cuyos documentos aluden a los primeros proyectos de iluminación por gas en España en las dos primeras décadas del siglo XIX; y diferentes obras de referencia para obtener una visión panorámica de los personajes estudiados, tales como el Biographical Dictionary of Civil Engineers, o el Dictionary of National Biography, ambos accesibles en la British Library.

Todo ello sin dejar de lado la documentación de los archivos y bibliotecas franceses y españoles, donde parte de la saga se mantuvo activa hasta la segunda mitad del siglo XIX. Así pues, la documentación de la Bibliothèque National de France permite conocer la participación de Aaron Manby en la fundición de Cha- renton o la siderurgia de Le Creusot. Por su parte, los fondos del Archivo Histórico de la Oficina Española de Patentes y del Archivo Histórico de Sevilla ayudan a identificar algunas de las empresas y proyectos en los que se embarcó Edward O. Manby, en colaboración con otros ingenieros e inversores destacados de la industria del gas, como William Partington o Melitón Martín. Finalmente, en la Biblioteca Nacional de España se pueden consultar algunos opúsculos y panfletos explicativos de las ventajas de la iluminación por gas frente a otros procedimientos tradicionales. Todo ello, sin descuidar la prensa española, que se hizo eco del éxito o fracaso de las diferentes empresas en las que el capital de los Manby y otros accionistas del momento participó, en un intento por dinamizar la industria del país.

\section{EL INICIO DE UNA SAGA: AARON MANBY (1776- 1850), EL PATRIARCA}

El apellido de Manby aparece ligado al inicio del alumbrado por gas en la Europa de comienzos de la Revolución Industrial, en dos de las principales potencias que lideraron el cambio: Gran Bretaña y Francia, en el tránsito del siglo XVIII al siglo XIX, para llegar a España a mediados de esta última centuria. El fundador de la saga, Aaron Manby, nació en Albrighton, Shropshire, el 15 de noviembre de 1776; su padre era Aaron Manby, natural de Kingston, en Jamaica, y su madre era Jane Lane, natural de Bentley, en el condado de Yorkshire del Sur, en Inglaterra. La información sobre su vida es escasa hasta que alcanzó los 36 años; ${ }^{1}$ entonces, en el año 1812, aparece como socio gestor de la Horseley Coal and Iron Company de Tipton, en Staffordshire, dedicada a la actividad siderúrgica, cuya creación se remontaba a finales del siglo XVIII y que incluía minas de carbón, altos hornos y talleres de ingeniería; pese a dedicarse a esta actividad, se desconoce si Aaron Manby tuvo formación como ingeniero.

Fue este el sector al que dedicó la mayor parte de su vida, inicialmente en el ámbito de la producción y procesado del mineral de hierro. Desde muy pronto estuvo al frente de la compañía y, por ejemplo, obtuvo una patente para usar nuevos materiales para la fabricación de ladrillos, que habría de emplear en la construcción de los altos hornos de Wolverhampton, donde trabajó como maestro siderúrgico. Entre los diferentes proyectos en los que la Horseley Coal and Iron Company participó ha de destacarse también la construcción de un gran tanque de agua en los East India Docks, en Londres este, en 1814, y al año siguiente la edificación de un puente giratorio de acero. En 1816 la sociedad se anunciaba ya como proveedora de alum- 
brado de gas (Biographical Dictionary, ed. 2008, pp. 431-432). Gracias a su colaboración, la Horseley Coal and Iron Company se implicó en varias innovaciones técnicas. Por ejemplo, en 1813 Manby obtuvo su primera patente de un método para aprovechar la escoria sobrante de los altos hornos en la fabricación de ladrillos y bloques para la construcción (Oxford Dictionary, ed. 2020). ${ }^{2}$

Las primeras experiencias de alumbrado de gas se habían desarrollado en Europa unas décadas antes, cuando comenzó la Revolución Industrial. William Murdoch fue el pionero de este sector industrial en el Reino Unido en 1792, con la iluminación de su casa particular, honor que correspondió a Philippe Lebon en Francia en 1799; sin embargo, aún se trataba de experimentos domésticos con medios rudimentarios. Fue el germano Friedrich Albrect Winzer, que después cambió su nombre por el de Frederick Winsor, quien inspirándose en Lebon apoyó la construcción de grandes fábricas centralizadas para dar servicio a varios establecimientos, mientras que Murdoch era partidario de emplear pequeñas fábricas para iluminar un establecimiento concreto. Winsor tenía además mayor visión empresarial que Murdoch, lo cual le llevó a adelantársele y solicitar una patente para la obtención de gas a partir del carbón. En 1812 fundó Gas Light and Coke Company, la primera sociedad del continente que suministró gas de manera regular (Thomas, 2014, pp. 4-6).

La mayor parte de la actividad ingeniera y empresarial de Aaron Manby no se desarrolló en su país de origen, sino en Francia. Habría obrado en su ánimo la convicción de que el escenario francés, en el que la Revolución Industrial se había iniciado con retraso respecto al Reino Unido, ofrecía mejores perspectivas de crecimiento y una menor competencia (Raveux, 1994, pp. 143-161). En 1819 se interesó ya en la iluminación de gas en territorio galo, comisionando a Daniel WiIson, químico natural de Dublín, para que presentara un proyecto destinado a obtener la concesión del alumbrado de París. El 12 de mayo de 1821 ambos, en colaboración con otro socio francés, Henry, se hicieron con la patente para la fabricación de gas, y en 1822 constituyeron la Compagnie d'éclarage par le gaz hydrogène, que seguía la estela de la compañía fundada por el propio Wilson un año atrás. El proyecto sufrió varios retrasos por la oposición de los detractores de este tipo de alumbrado, pero los socios no se arredraron y consiguieron construir otras fábricas de gas en Les Ternes en 1825; cuatro años después, la conocida como la Compagnie Anglaise dio un importante paso al alumbrar con gas la Rue de la Paix, en París. Manby amplió su radio de acción y también invirtió en el alumbrado de Hannover y Berlín, en 1825 y 1826, respectivamente (Chaloner y Henderson, 1995, pp. 63-75).

Los otros industriales en los que destacó fueron la siderurgia y la construcción naval. En 1821 estableció una siderurgia operada con gas en Charenton, cerca de París, nuevamente con la inestimable colaboración de Wilson. ${ }^{3}$ Este había hecho valer sus conocimientos sobre química para obtener una patente para usar amoniaco en la separación del sulfuro de hidrógeno del gas (The Repertory, 1818, pp. 11-16). La citada siderurgia de Charenton tuvo tal repercusión en la economía industrial francesa que la Société d'Encouragement pour I'Industrie National concedió a sus fundadores la medalla de oro. En aquel momento Manby y Wilson empleaban alrededor de 500 trabajadores y habían contribuido decisivamente a romper la dependencia del país galo de ingenieros británicos. Esto no agradó a sus compatriotas, quienes primero le acusaron de llevarse mano de obra nacional al extranjero (Robinson, 2009), para en 1824 denunciarle ante la comisión parlamentaria de artesanos y maquinaria (Oxford Dictionary, ed. 2020). ${ }^{4}$

Aaron Manby expandió la actividad, haciéndose en 1826 con la propiedad de la siderurgia de Le Creusot, que tanto él como Wilson modernizaron con nuevas técnicas y equipamiento también renovado. ${ }^{5}$ Aquí se fabricaron, por ejemplo, los raíles para el ferrocarril de St. Etienne-Lyon, y parte de estas innovaciones se presentaron en la Exposición Industrial de París de 1827. Al año siguiente, Manby y Wilson fusionaron ambas siderurgias en la Société Anonyme des Mines, Forges et Fonderies du Creusot et de Charenton, si bien para aquella fecha los altos hornos de Charenton habían quebrado ya. La crisis financiera de 1830-1831 les golpeó con dureza, no pudiendo beneficiarse de los $2 \mathrm{mi}$ llones de francos que el gobierno francés ofreció a las empresas para salir de aquella coyuntura, pues los dos créditos que solicitaron, de 500.000 francos primero y de 250.000 después, les fueron denegados. En 1833 la Société se declaró en bancarrota, con una deuda superior a los 11 millones de francos, hasta que en 1836 sus acreedores vendieron Le Creusot a los hermanos Schneider, por una suma total de 2.680 .000 francos (Chaloner y Henderson, 1955, pp. 63-75). Eso no debe mover a desestimar la importancia de la actividad empresarial desarrollada por Manby y Wilson (Payen, 1969, pp. 104-105; Cotte, 2010, pp. 119-130).

En el ámbito de la construcción naval, en 1819 Aaron Manby se había asociado con el capitán Charles Napier, más tarde vice-almirante, que en aquella fecha 
viajó a París para fundar la Société des Transports Accélérés par Eau. Fue entonces cuando comenzaron a trabajar en el proyecto de un barco de vapor fabricado íntegramente en hierro, para lo cual Aaron solicitó en 1821 una patente de construcción de un motor marino de oscilación (Chatwin, 1972, pp. 10-35; Biographical Dictionary, ed. 2008, pp. 431-432). En honor a su inventor, este barco, completado el 30 de abril de 1822, recibió el nombre de Aaron Manby. ${ }^{6}$ Fabricado en los altos hornos de Horseley, se trasladó por piezas al muelle de Surrey, donde se ensambló y se botó en el río Támesis el día 9 de mayo. Pocos días después salió desde Londres, comandado por Napier, cargando mercancías que llegaron a Boulogne el 18, desde donde continuó ruta hasta París. ${ }^{7}$

Sus años finales fueron discretos: tras la crisis de la década de 1830 decidió regresar a Gran Bretaña. Después de residir brevemente en Fulham y en Ryde, se mudó a Shanking, en la Isla de Wight, de la cual era originario, donde murió el 1 de diciembre de 1850. En vida contrajo matrimonio dos veces: la primera con Julia Fewster, que dio a luz a su primogénito y principal socio, Charles; la segunda con Sarah Haskins, con quien tuvo una hija y tres hijos: John Richard, Joseph Lane y Edward Oliver, los dos últimos muy activos en España.

\section{LA SAGA MANBY, PUENTE DESDE GRAN BRETAÑA AL CONTINENTE: FRANCIA Y ESPAÑA}

El objetivo aquí es analizar la trayectoria profesional de tres de los cinco hijos de Aaron Manby: Charles (1804-1884), Joseph Lane (1814-1862) y Edward Oliver (1816-1864). El primogénito acabó sucediéndole en los negocios familiares del Reino Unido y Francia tras su muerte. Por su parte, Joseph Lane y Edward Oliver trascendieron ambas fronteras para desarrollar su actividad emprendedora e industrial en España, solicitando patentes de invención industrial e impulsando el desarrollo del alumbrado por gas.

En la siguiente tabla se presenta un resumen de la actividad desarrollada por todos los integrantes de la saga:

\subsection{Charles Manby (1804-1884), Seguidor De LA ESTELA PATERNA}

Al principio la carrera de Charles se enfocó hacia el Ejército: su tío, el capitán Joseph Manby, ocupaba un lugar distinguido entre el personal de servicio del duque de Kent, lo cual aprovechó para obtener para Charles una comisión que le permitiese desarrollar parte de su formación en la academia de Saint Servan, cerca de Rennes, donde llegó en 1814. Su arribada a Francia coincidió con el Imperio de los Cien Días y la derrota definitiva de Napoleón Bonaparte en Waterloo (1815). Convencido de que el final de las Guerras Napoleónicas comprometía las posibilidades de ascenso de su hijo, Aaron Manby ordenó su regreso al Reino Unido, donde se formó en ciencias y matemáticas, probablemente con profesores particulares.

Desde 1817 se convirtió en el principal socio paterno. Gracias a la posición de su progenitor, Charles aprendió ingeniería de manera práctica, en la Horseley Ironworks, antes de dar el salto a Francia en 1819. Tres años después el patriarca de la familia rompió sus vínculos con aquella empresa, por lo que reclamó en el país galo a varios trabajadores que habían estado a su servicio, entre ellos su hijo Charles. El primer puesto que ejerció fue el de colaborador de David Wilson en la fundición de Charenton, antes de jugar el papel de encabezar la modernización de la siderurgia de Le Creusot. Entre 1826 y 1829 su actividad al servicio de la economía y la industria francesas llegaron a ser tan relevantes que el gobierno galo le nombró ingeniero del Departamento de Tabaco, encargándole la construcción de fábricas de esta mercancía por todo el país. Un nuevo reconocimiento llegó también de la mano del mariscal Soult, quien le otorgó una condecoración militar.

El año de 1829 marcó el final de su periplo francés y regresó al Reino Unido, donde se hizo cargo de la siderurgia de Beaufort. Entonces conoció a la que sería su esposa, Ellen Jones, con quien se casó al año siguiente. A comienzos de la década de 1830 focalizó sus esfuerzos en el desarrollo del ferrocarril en Gales del Sur, como miembro de la Ebbw Vale Iron Co., y contribuyó al avance de la industria siderúrgica en el puerto de Bristol. En 1835 se trasladó a Londres, donde se estableció como ingeniero civil independiente acometiendo, entre otros, un proyecto para la ventilación de los edificios. Tres años después se le nombró ingeniero de una compañía creada por Sir John Ross que fletaba barcos de vapor en Londres. Poco después la adquirió la Peninsular \& Oriental Steam Company, que abrió una ruta comercial hacia la India (Lalvani, 2016, p 77).

Ya en 1839 resultó electo como miembro de la Institución de Ingenieros Civiles (Biographical Dictionary, ed. 2008, pp. 431-432). Entre sus labores más destacadas ha de mencionarse su participación, como miembro y secretario, en la Comisión Científica Internacional para el Canal de Suez (Lesseps, 1856, pp. 266-339; Chaloner \& Henderson, 1955, pp. 63-75). ${ }^{8}$ De igual forma, merece especial atención su asesora- 
Tabla 1. Integrantes y actividades empresariales de la saga de los Manby

\begin{tabular}{|c|c|c|}
\hline Nombre y apellidos & $\begin{array}{l}\text { Año de la } \\
\text { iniciativa }\end{array}$ & Empresa en la que participaron \\
\hline \multirow{17}{*}{ Aaron Manby (1776-1850) } & 1812 & Horseley Coal and Iron Company (Tipton -Staffordshire). \\
\hline & (No figura) & Construcción de los altos hornos de Wolverhampton. Ejerce como maestro siderúrgido. \\
\hline & 1814 & Construcción de un tanque de agua en los East India Docks de Londres. \\
\hline & 1815 & Construcción de un puente giratorio de acero. \\
\hline & 1816 & La Horseley Coal and Iron Company se anuncia como proveedora de alumbrado de gas. \\
\hline & 1819 & Société des Transports Accélérés par Eau. \\
\hline & 1821 & Siderurgia operada con gas en Charenton. \\
\hline & 1822 & Compagnie d'éclarage par le gaz hydrogène. \\
\hline & 1822 & Construcción del Aaron Manby, barco de vapor fabricado íntegramente en hierro. \\
\hline & 1825 & Fábricas de gas en Les Termes. \\
\hline & 1825 & Alumbrado de Hannover. \\
\hline & 1826 & Alumbra de Berlín. \\
\hline & 1826 & Le Creusot. \\
\hline & 1827 & $\begin{array}{l}\text { Fusión de sus dos empresas siderúrgicas (Charenton y Le Creusot) en la Société } \\
\text { Anonyme des Mines, Forges et Fonderies du Creusot et de Charenton. }\end{array}$ \\
\hline & 1814 & Formación en la Academia de Saint Servan. \\
\hline & 1817 & Asociado de su padre en la Horseley Ironworks (Horseley Coal and Iron Company). \\
\hline & 1819 & Colaborador de su padre y de David Wilson en Charenton y Le Creusot. \\
\hline \multirow{11}{*}{ Charles Manby 1812} & 1814 & Formación en la Academia de Saint Servan. \\
\hline & 1817 & Asociado de su padre en la Horseley Ironworks (Horseley Coal and Iron Company). \\
\hline & 1819 & Colaborador de su padre y de David Wilson en Charenton y Le Creusot. \\
\hline & $1826-1829$ & $\begin{array}{l}\text { Ingenierio del Departamento de Tabaco del gobierno de Francia; } \\
\text { encargado de construir fábricas de tabaco por todo el país. }\end{array}$ \\
\hline & 1829 & Siderurgia de Beaufort. \\
\hline & $1830 \mathrm{~s}$ & Construcción del ferrocarril en Gales del Sur en la Ebbw Vale Iron Co. \\
\hline & $1830 \mathrm{~s}$ & Desarrollo del sector en la ciudad portuaria de Bristol. \\
\hline & 1835 & Ingeniero independiente establecido en Londres. \\
\hline & 1838 & $\begin{array}{l}\text { Ingeniero de la compañía presidida por Sir John Ross } \\
\text { que fletaba barcos de vapor en Londres. }\end{array}$ \\
\hline & (No figura) & $\begin{array}{l}\text { Propietario de la Peninsular \& Oriental Steam Company, } \\
\text { que abre una ruta comercial hacia la India. }\end{array}$ \\
\hline & 1839 & $\begin{array}{l}\text { Miembro de la Institución de Ingenieros Civiles. Participa, en calidad de miembro } \\
\text { y secretario, en la Comisión Científica Internacional para el Canal de Suez. }\end{array}$ \\
\hline \multirow{7}{*}{ Joseph Lane Manby (1814-1862) } & $1830 \mathrm{~s}$ & Trabajo en la Compagnie Anglaise, fundada por su padre y David Wilson en Francia. \\
\hline & $1830 \mathrm{~s}$ & Gestión de la Continental Gas Company en Francia. \\
\hline & 1852 & $\begin{array}{l}\text { Desarrollo de un proyecto de línea de ferrocarril entre } \\
\text { Córdoba y Sevilla junto a su hermano. }\end{array}$ \\
\hline & $1830 \mathrm{~s}$ & Colabora con su hermano Charles en la Ebbw Vale Iron Works. \\
\hline & $1830 \mathrm{~s}$ & Construcción de los altos hornos de Swansea. \\
\hline & $1830 \mathrm{~s}$ & Ingeniero independiente en Swansea y Londres junto a su hermano John. \\
\hline & 1846 & Sociedad Madrileña de Alumbrado de Gas. \\
\hline \multirow{7}{*}{ Edward Oliver Manby (1816-1864) } & 18461 & $\begin{array}{l}1846 \text { Empresa General Peninsular del Alumbrado por Gas (responsable } \\
\text { de la fábrica de gas de Cádiz a finales de la década dd 1840). }\end{array}$ \\
\hline & 1849 & Desarrollo del tramo Madrid-Segovia. \\
\hline & 1851 & Abastecimiento de agua de Madrid desde la sierra de Guadarrama. \\
\hline & 1852 & $\begin{array}{l}\text { Desarrollo de un proyecto de línea de ferrocarril entre } \\
\text { Córdoba y Sevilla junto a su hermano. }\end{array}$ \\
\hline & 1855 & Línea entre la Roda y el criadero carbonífero de Hinajeros. \\
\hline & 1855 & Proyecto de ampliación de la Puerta del Sol en Madrid. \\
\hline & 1856 & Línea entre Madrid y Lisboa. \\
\hline
\end{tabular}


miento en la creación de los cuerpos de personal de ingenieros y voluntarios ferroviarios. En los siguientes años su actividad fue decayendo hasta su muerte sin descendencia el 21 de julio de 1884 en Londres (Oxford Dictionary, ed. 2020). ${ }^{9}$

\subsection{JOSEPH LANE (1814-1862) Y EDWARD OLIVER (1816- 1864) MANBY: EL SALTO A ESPAÑA}

El destino de los dos hermanos cuya biografía aquí se acomete estuvo entrelazado en diferentes momentos de su vida, por lo que en las siguientes páginas se aborda el análisis de ambos personajes de manera conjunta, subrayando las etapas vitales en las cuales sus intereses confluyeron, con especial atención a los años transcurridos en España.

\section{Infancia y juventud}

Joseph Lane Manby nació en Horseley, Staffordshire, el 22 de diciembre de 1814; su hermano Edward Oliver Manby lo haría un año y medio después, el 17 de junio de 1816. En 1820, cuando apenas contaban seis y cuatro años, respectivamente, los dos se trasladaron a Francia, donde el patriarca acababa de embarcarse en la creación de la Société des Transports Accélérés par Eau (Chatwin, 1972, pp. 10-35; Biographical Dictionary, ed. 2008, pp. 431-432), para después participar también en la siderurgia gala con la explotación de los altos hornos de Charenton y Le Creusot (Cotte, 2010, pp. 119-130; Oxford Dictionary, ed. 2020). ${ }^{10}$

Allí realizaron sus estudios primarios en la prestigiosa escuela de Massin ("Élements biographiques", 1866, pp. 187-189). Después sus caminos se separaron: Joseph inició su formación como ingeniero en la fábrica de la Compagnie Anglaise que habían constituido David Wilson y su padre (Chaloner y Henderson, 1995, pp. 63-75). De este modo, igual que Aaron Manby y otros ingenieros formados en el extranjero, adquirió sus conocimientos técnicos de manera práctica, trabajando en las fábricas. Por su parte, Edward marchó a la Universidad de Gante, en la cual cursó estudios de química y matemáticas, graduándose con honores en 1832. Ambos estaban ya listos para comenzar su vida profesional, que Joseph habría de desarrollar de vuelta en el Reino Unido, como empleado de la firma de Jeremiah Harman \& Co., en Londres ("Obituary", 1863, pp. 629-630).

Edward también regresó a su país natal, en su caso para ampararse en la tutela de su hermano mayor Charles, quien se apoyó en él para la gestión de la Ebbw Vale Iron Works. Sus conocimientos de química fueron útiles para introducir la antracita en el proceso de fundición. Conocedor de los avances técnicos apli- cados en esta compañía, el empresario Crane, natural de Ynischedwyn, cerca de Swansea, requirió sus servicios para la construcción de los altos hornos, donde también experimentó con nuevas y exitosas técnicas. Gracias al prestigio que consiguió labrarse, se sintió movido a establecerse como ingeniero independiente en Swansea, donde se le unió brevemente su hermano menor John. Desde Swansea pasaron a Londres y, finalmente, se establecieron en Madrid, que ofrecía grandes oportunidades, habida cuenta del estadio aún germinal de la industria española ("Obituary", 1865, pp. 533-534).

En este nuevo destino se les uniría pronto Joseph, quien tras la quiebra y disolución de la firma de Jeremiah Harman \& Co. siguió el consejo de George Lowe, amigo de su padre, y se trasladó a Francia, donde se hizo cargo de la gestión de la Continental Gas Company ("Obituary", 1863, pp. 629-630). Después de esta nueva experiencia en suelo francés marchó a España junto a sus hermanos Edward y John, donde en adelante abarcarían un amplio abanico de actividades vinculadas con la siderurgia, el sector ferroviario, la iluminación por gas, y otras empresas como la conducción de aguas (Heredia-Flores, 2013, pp. 103-117).

\section{El despegue de la industria del gas en España: la sociedad entre Edward Oliver Manby y William Partington Hurts}

Mediada la década de 1840, los hermanos Joseph y Edward Manby están ya en España, concretamente en Madrid, para pilotar el despegue industrial del país (Raveux, 1994, pp. 143-161; Arroyo, 2000, pp. 45-72; Tortella, 2000, p. 54). Los primeros ensayos de alumbrado de gas en España se registraron en Granada y Cádiz en 1807 y en Alcoy en 1817 (García de la Fuente, 1990; 1998, pp. 62-64; 2002, pp. 161-163; Fernández-Paradas, 2015, p. 24). La documentación demuestra que en Cádiz el capital británico ya se había interesado en 1824 en invertir en el alumbrado de la ciudad por gas: concretamente, el inventor Sir WiIliam Congreve solicitó aquel año al cónsul británico del lugar, Sir John McPherson Brackenbury, información sobre la disposición del ayuntamiento a aceptar este sistema de alumbrado. La respuesta del cónsul era descorazonadora: en aquel momento Cádiz disponía de 941 lámparas, además de 17 concentradas en la Plaza de San Antonio, iluminadas por aceite. Como el aceite, indicaba el cónsul, era tan barato, el costo anual del alumbrado, incluyendo el precio del aceite, era de unos 15.000 dólares, debiendo abonar cada distrito 100 dólares mensuales.

MacPherson coincidía con Congreve en que la incorporación del gas permitiría iluminar la ciudad me- 
jor y con menos lámparas, que proporcionarían una luz más brillante. Las ventajas del nuevo sistema de alumbrado eran bastante claras, pero existían tres obstáculos fundamentales, a su juicio: primeramente, el estado de zozobra en el que se hallaba España, con el absolutismo recién restaurado por Fernando VII e inmersa en un duro proceso de represión de los liberales que habían apoyado al régimen constitucional previo (1820-1823); en segundo lugar, la compleja coyuntura exterior del país, afectado por la pérdida de sus colonias de Hispanoamérica, que había perjudicado seriamente tanto a España como al puerto de Cádiz; finalmente, el carácter español: ni el ayuntamiento de Cádiz ni ningún otro aceptaría el nuevo sistema de alumbrado por gas salvo que recibiera a cambio un beneficio claro por ello. Lo mismo, concluía el cónsul en su misiva a Congreve, sucedía con el rey, quien únicamente aceptaría oír el proyecto a cambio de alguna prebenda: "I dare say before His Catholic Majesty would condescend to give his royal sanction to the lighting of all his cities in Spain with gas, he would expect a few ounces of gold and a few boxes of cigars by way of douceur". ${ }^{11}$

Barcelona fue pionera en la inauguración de instalaciones de alumbrado con gas, cuando en 1826 se estrenó la iluminación del aula de dibujo de la Escuela de Nobles Artes, en la Casa Lonja, con el fin de iluminar las clases nocturnas; un año más tarde se hacía lo propio en la Escuela de Bellas Artes, en presencia del propio Fernando VII (Sudrià, 1983, p. 103; Fàbregas, 1993; Simón Palmer, 2011, pp. 15-16). Como era de esperar, la siguiente ciudad en incorporar el nuevo sistema de alumbrado fue Madrid, procediendo el ayuntamiento a convocar un concurso para instalar el alumbrado público de gas. Como el consistorio careciera de fondos suficientes para sufragar por sí solo los primeros proyectos que se le presentaron, en las décadas de 1820 y 1830 las iniciativas se limitaron al entorno privado. Tras una primera iluminación efímera en algunas calles de la capital a cargo de José Roura, con motivo del nacimiento de la infanta Luisa Fernanda (segunda hija de Fernando VII y María Cristina), se construyó la Fábrica del Palacio Real, inaugurada en 1833 con ocasión del nombramiento de la infanta Isabel como Princesa de Asturias y del nacimiento de su hermana Luisa Fernanda (Fàbregas, 2017, pp. 21-44). Al principio, todas estas instalaciones empleaban idénticos medios para producir el gas, a partir de la quema de carbón en retortas de mampostería mediante fuego directo (Alayo Manubens \& Barca Salom, 2017, pp. 141-171).

La incorporación de métodos más novedosos, unida a la generalización del uso del gas tanto en el ámbito privado como en el espacio público, vino de la mano de Edward O. Manby y su principal socio colaborador en estos años: William Partington Hurts (Fernández-Paradas \& Pinto Tortosa, 2020, pp. 349-365). El año 1846 sería el del despegue del alumbrado de gas público en la capital, cuando ambos socios constituyeron la Sociedad Madrileña de Alumbrado de Gas, con el apoyo de José de Salamanca, el marqués de Salamanca (Fàbregas, 2017, pp. 21-44). En febrero de aquel mismo año obtuvieron la concesión de los faroles públicos de la capital, hasta entonces de la propiedad de los hermanos Gil y Serra. El retraso en el desarrollo de este nuevo sistema de alumbrado en España, con un desfase de dos décadas respecto a Gran Bretaña y Francia se explica por la combinación de tres factores: el obstáculo que representaban los privilegios gremiales, que subsistieron hasta 1834; la ausencia de bancos en sentido moderno hasta 1844; y la carencia de ferrocarril hasta 1848.

La industria del gas fue la primera industria pesada en el país, gracias a la habilidad de Manby y Partington, que supieron sortear los obstáculos hallados por los promotores previos, a saber: la escasa previsión sobre las inversiones requeridas, un espíritu emprendedor en vías de desarrollo y el empeño del ayuntamiento de Madrid en que solo se emplease utillaje fabricado en España. Su virtud residió en la capacidad de argumentar ante el consistorio la mejor forma de fabricar gas, basándose en experiencias como la ocurrida en Courcelles, en París, en 1824, que Edward Manby conocía de primera mano. Recurriendo a este ejemplo, acabaron persuadiendo a las autoridades municipales de la necesidad de producir gas a partir de carbón, mediante el uso de materiales de fuera. Se dejaba así de lado otros proyectos, como el de José Viejo Medrano, consistente en obtener gas a partir de aceite de oliva. En aras de obtener mayor respaldo popular al nuevo sistema de alumbrado, publicaron la Memoria sobre el Alumbrado de Gas de la Villa de Madrid. En ella, además de subrayar las ventajas técnicas de este sistema de iluminación, anunciaban el apoyo de los primeros capitalistas españoles, que habían hecho posible que la Sociedad Madrileña iniciara su andadura con un capital inicial de 12 millones de reales, divididos en 3.000 acciones de 4.000 reales cada una (Manby \& Partington, 1846)..$^{12}$

El objetivo de Manby y Partington era crear una fábrica para abastecer 1.000 farolas públicas y 5.000 instalaciones privadas en la capital del reino. Más adelante pretendían alcanzar la cifra de 10.000 luces públicas, que abastecerían con una canalización subterránea de 20.750 metros de radio. Apenas tres meses después decidieron constituir la Empresa General Peninsular 
del Alumbrado por Gas, también con el patrocinio del marqués de Salamanca, con un capital social inicial de 50 millones de reales, distribuidos en 25.000 acciones de 2.000 reales cada una (Fernández-Paradas, 2009). ${ }^{13}$ Interesa el carácter integrador con el que los dos empresarios británicos concibieron La Peninsular, pues en sus estatutos se decía explícitamente que también desarrollaría otras industrias y sectores asociados, como la minería de carbón y de hierro, así como la siderurgia para la fabricación de los conductos subterráneos y la maquinaria de las fábricas. Ahora bien, en estos primeros años el suministro de materia prima vino del exterior, convirtiéndose pronto en un foco de conflicto para los impulsores de la Sociedad Madrileña y de La Peninsular.

El 5 de diciembre de 1846 los dos firmaron un contrato con Frederick Deeley y Thomas Thomas, quienes crearon el negocio Iron Founders en el condado de Stafford, bajo la firma Deely \& Thomas, comprometiéndose a suministrar a Manby y Partington los materiales necesarios, que los compradores podían inspeccionar personalmente in situ cuando así lo desearan. En marzo de 1847 se procedió al envío de la primera remesa, desde Gloucester al puerto de Málaga, pero a mediados de abril Manby y Partington pidieron a la Deeley \& Thomas el cese inmediato de la producción para modificar las cantidades y el tipo de tuberías que requerían.

Los fabricantes estuvieron de acuerdo, anunciando que el cambio implicaría sobrecostes, pero fue en este punto donde se produjo el choque entre ambas partes, por lo que el letrado Jones, empleado de los dos socios, exigió el 28 de abril el cese inmediato de toda producción en nombre de sus representados. Entonces se inició un lento proceso judicial en el que se sucedió la demanda de los sobrecostes correspondientes por parte de la compañía demandante, frente a las constantes declaraciones de desacuerdo de los demandados. Aunque la quiebra de la Sociedad Madrileña en 1849 podría haber zanjado la cuestión, no fue así y tanto Manby como Partington ofrecieron a Deeley y Thomas promisorias por un valor total de 1.165 libras en la Compañía para el Alumbrado de Gas de Madrid, pero el alivio económico fue nulo para los demandantes (de hecho, en aquel mismo año Thomas entró en bancarrota). Deseoso de una reparación, en 1851 Deeley solicitó el arresto de Partington, entonces en un viaje de negocios en el Reino Unido, que se produjo el 1 de abril del año siguiente. Un día después se le permitió volver a España, bajo la promesa de consultar los libros de cuentas de la antigua Sociedad Madrileña para satisfacer los requerimientos de sus antiguos proveedores, pero en la práctica fue incapaz de satisfacer la deuda. El 22 de mayo de 1858 la Deeley \& Thomas renunció a cualquier reclamación contra Manby y Partington. ${ }^{14}$

De la mano de La Peninsular, los dos británicos acometieron el alumbrado de gas de diferentes capitales españolas, entre las que cabe destacar los casos de Málaga y Sevilla. En la primera, en 1846 Partington obtuvo la contrata, traspasándola pocos meses después a La Peninsular (Arroyo, 2001, pp. 297-325). Actuaban así según el patrón de conducta común en el sector del gas en este momento (Fernández-Paradas \& Pinto Tortosa, 2020): tras obtener la concesión en una población, la revendían a otra sociedad y beneficiarse del sobrecoste de la reventa. No obstante, La Peninsular fue incapaz de acometer el servicio de alumbrado público por gas en la capital malagueña, y el negocio pasó en 1852 a manos de Louis-Jean Gosse. También en 1846 iniciaron los trámites para obtener la concesión en Sevilla, recurriendo al ingeniero polaco Karl Karsnicki como apoderado y representante. Además de la contrata adquirieron "el Perneo", en las afueras de la ciudad, en régimen de arrendamiento durante 25 años para construir la fábrica de gas, comprometiéndose a pagar 3.000 reales de renta por adelantado. Sin embargo, a finales de ese mismo año acabaron reconociendo su imposibilidad de acometer el servicio ni de pagar la renta del terreno, por lo que en 1852 la propiedad de la contrata pasó a manos de Jaime Federico Gregory. ${ }^{15}$

Cádiz, Valencia ${ }^{16}$ o Santander fueron otros escenarios donde intentaron desarrollar su actividad, con idéntico resultado, de modo que en apenas unos años se frustraron sus proyectos de hacer fortuna en el sector del gas en España, al mismo tiempo que se consolidaba su imagen más como "conseguidores" de concesiones que como explotadores. La Sociedad Madrileña experimentó complicaciones desde muy pronto, perjudicada por la dificultad de obtener carbón a precio bajo y por el inicio de un ciclo económico negativo (Fernández-Paradas, 2009, p. 32). Este factor condicionó a los socios y accionistas, que en febrero de 1847 comenzaron a incumplir el pago de su parte de la empresa, y en septiembre los dueños debieron advertir de que, a menos que dichos pagos se hicieran a tiempo, habría que vender las acciones a bajo precio e incluso cesar la actividad. ${ }^{17}$ Pese a las reclamaciones, un año después la Sociedad Madrileña se hallaba próxima a la quiebra, por lo cual sus dueños nombraron al ingeniero Melitón Martín Arranz como director técnico, puesto que conservó hasta 1853, permaneciendo como socio de la compañía hasta 1856, cuando quebró (García Hourcade, 2010, p. 27; Simón 
Palmer, 2011, pp. 71, 78; Fernández-Paradas \& Pinto Tortosa, 2019).

También en 1856 La Peninsular se declaró en quiebra y liquidada, pese a las objeciones de Partington y Manby, quedando desde entonces bajo el control de capital francés a través de la Compañía Madrileña de Alumbrado y Calefacción por Gas (Simón Palmer, 1989, pp. 73, 81; Fernández-Paradas, 2019, p. 111; Fernández-Paradas, Larrinaga \& Matés Barco, 2021). A pesar de su fracaso, el destino del alumbrado de gas en España no siguió el mismo camino, puesto que otros individuos desarrollaron el servicio en el futuro, con éxito desigual. Ha de reconocérseles el mérito de haber sido pioneros en la introducción de este sistema de iluminación, que a la postre constituyó uno de los pilares esenciales del despegue industrial español. Edward Manby empleó estas razones cuando en 1849 se defendió de los ataques que le dirigió Vicente Calderón desde las páginas de El Heraldo, editado por el hermano de este. Manby esgrimió varios argumentos: la mayor rentabilidad del gas, pues producía mayores beneficios con menores ingresos, y su contribución al despegue de otros sectores industriales. ${ }^{18}$

\section{Joseph y Edward Manby como inversores en otras actividades y sectores industriales: la diversificación del riesgo}

Coincidiendo con el declive de los proyectos gasísticos de Edward, Joseph Manby desarrolló una breve pero intensa actividad profesional en España en el ámbito de la ingeniería, que conocemos gracias a las patentes de invención que solicitó. Primeramente, el 23 de marzo de 1849 pidió un nuevo certificado de introducción para la fabricación de sustancias espirituosas destinadas a alumbrar, a calentar y a otros usos, conforme a un procedimiento que se utilizaba ya en Inglaterra. La resolución de la reina fue favorable, previo pago de 3.000 reales, y comenzó a prestar sus servicios conforme a lo acordado. ${ }^{19}$ El 2 de junio de 1849 presentó una solicitud de certificado de introducción de un procedimiento para la fabricación de pan mediante hornos continuos caldeados con aire caliente. Suscribieron la solicitud con él Mariano Sigler, vecino de Valladolid, y José Ruiz de Quevedo, vecino de Madrid. En la exposición de motivos se insistía en que el procedimiento era más ventajoso que los empleados hasta la fecha pues permitía economizar en mano de obra y combustible, asegurando la limpieza perfecta del horno y la cocción regular. La reina resolvió nuevamente a su favor a cambio del pago de otros 3.000 reales, y un año más tarde el nuevo procedimiento se había puesto ya en marcha en Valladolid con éxito. ${ }^{20}$
Obtuvo una resolución favorable más a su solicitud de certificado de introducción de un procedimiento para cocer ladrillos, tejas y otras materias mediante hornos Ainslie, que ya había utilizado antes en su país natal, y que data de 7 de marzo de 1850. Exigiendo las mismas condiciones que en las solicitudes anteriores, la Corona resolvió positivamente. ${ }^{21}$ En noviembre de este mismo año cursó una solicitud de privilegio de invención para un nuevo método que permitiera separar los minerales de sus gangas, ${ }^{22}$ y en enero de 1851 hizo lo propio para la destilación del carbón de piedra y otras sustancias empleadas en la fabricación de gas. ${ }^{23}$ En ninguno de los dos casos existe constancia ya de la aprobación por parte de Isabel II, circunstancia que debió coincidir con su marcha a París, donde se asoció con Monsieur Savalète, con quien se embarcó en adelante en varios proyectos inmobiliarios ("Obituary", 1863, pp. 629-630). Entre ellos ha de destacarse la construcción ferroviaria en España, que constituyó un modo de mantener su vínculo con el país en el que su hermano Edward aún seguía residiendo.

Edward había participado ya en 1849 en el proyecto para construir la línea entre Madrid y Segovia, cuya finalización, estimada en cuatro años en ambos casos, era fundamental para el buen abastecimiento de la capital. ${ }^{24}$ Fue en 1852 cuando los dos hermanos colaboraron en el proyecto para el ferrocarril que debía unir Córdoba y Sevilla, elevando desde esta ciudad al gobierno un informe sobre las ventajas que reportaría el trazado ferroviario para la región. El plan que remitieron fue bastante detallado, pues incluía no solo la previsión de capital que debería invertirse para su ejecución, sino también un desglose de los datos de explotación, así como la propuesta de tarifa de viaje para pasajeros, animales y mercancías, para que la explotación resultara rentable. Su proyecto es interesante porque en el plano, que adjuntaron a la exposición al ejecutivo, se apreciaba que la línea atravesaba las minas de carbón de Espiel y Bélmez, en Córdoba, con dos importantes ramales, uno a Écija y otro al Pedroso: ${ }^{25}$ Melitón Martín Arranz retomaría tiempo después el proyecto de los hermanos Manby desde el Congreso, en el que gozó de un escaño de diputado en $1861 .{ }^{26}$

Un año después el propio Martín estaría a cargo del proyecto de construcción de la línea ferroviaria, que acabó abandonando pronto (Torquemada Daza, 2006, pp. 1-26; Fernández-Paradas \& Pinto Tortosa, 2019). Aún participaría Edward Manby en la década de 1850 en sendos proyectos ferroviarios en Cuenca, para estudiar la línea que uniría la Roda con el criadero carbonífero de Hinajeros; ${ }^{27}$ y en la línea de Madrid a Lisboa. ${ }^{28}$ Como otros inversores y emprendedores de 
su época, se embarcó en algunas empresas sobre canalización de aguas, lo cual lo señala como un individuo preocupado por fomentar todos los aspectos que, en sentido amplio, favorecen el progreso de los pueblos (Fernández-Paradas \& Pinto Tortosa, 2019). Entre ellos, subrayamos el plan de abastecimiento de aguas de Madrid desde el Guadarrama, frente al proyecto previo de Juan de Rivera que aspiraba a emplear el agua del río Lozoya: la mayor proximidad de la sierra respecto a la capital, la existencia de algunas obras de ingeniería previas de la época de Carlos III, y finalmente la posibilidad de extraer aguas del lecho del río y conducirlas en tubos de mampostería, hacían el proyecto de Manby más rentable. ${ }^{29}$

La última empresa destacable en la que participó fue la presentación en 1855 de un plan para llevar a cabo el ensanche de la Puerta del Sol de Madrid, que ya se había intentado renovar en 1854, quedando los trabajos interrumpidos. Su estudio, presentado conjuntamente con el conde de Hamal, era más rentable y orgánico que el de su principal opositor, José Antonio Font. ${ }^{30}$ Los dos socios se comprometían a sufragar los gastos de la obra y pagar la indemnización correspondiente a los dueños de los locales expropiados. No obstante, pese a contar con la aprobación de la Academia de San Fernando, el plan se acabó rechazando y se acometió la reforma proyectada por Font. Concluida esta empresa, a finales de la década de 1850 volvió a París, donde continuó su actividad emprendedora e industrial junto a su hermano Joseph. Aquejado súbitamente de una grave enfermedad, falleció el 21 de febrero de 1864 con tan solo cuarenta y ocho años (“Obituary”, 1865, pp. 533-534).

\section{CONCLUSIONES}

La saga de los Manby suscita el interés del historiador porque representa una familia emprendedora en la cual la práctica totalidad de sus integrantes manifestaron el espíritu empresarial y la visión de oportunidad precisas para contribuir al despegue industrial de Europa. Todo ello sin haber recibido una formación universitaria necesariamente, pues las exigencias de la nueva era industrial demandaban un personal técnico capaz de formarse con rapidez en las propias fábricas. Así sucedió en los casos de Aaron Manby, cabeza de la familia, de su primogénito Charles y de Joseph Lane; Edward Oliver Manby fue la excepción, cursando estudios universitarios antes de aprender el oficio de ingeniero en las fábricas de su padre.
El éxito de las empresas que pusieron en marcha a lo largo de su vida no debe valorarse en función de su rendimiento económico: para obrar en justicia, es preciso contrapesar su balance de resultados con el impacto de sus iniciativas en el continente. En el caso de Aaron, el éxito inicial en los sectores siderúrgico y gasístico en el Reino Unido le movió a trasladarse a Francia, en busca de un mercado por explotar y, en consecuencia, con menor competitividad. Pese a que la explotación de los altos hornos de Charenton y Le Creusot no le reportó tantos beneficios como habría deseado, su contribución al despegue de la industria del gas aplicado a la iluminación, así como la pionera fabricación de un barco de hierro accionado por un motor de vapor, le convirtieron en uno de los individuos cuya contribución al desarrollo del sector secundario en aquel país había sido más destacada.

Lo mismo sucedió con sus hijos: Charles ejerció como primogénito, tutelando a sus hermanos y tomando el testigo de los negocios de su padre, con un balance en general bastante positivo. Por su parte, Joseph y Edward actuaron en tres escenarios nacionales distintos (Reino Unido, Francia y España), en los que desarrollaron una prolija actividad encaminada a reportarles beneficio, a la par que contribuían a favorecer la elevación de aquellos países a la categoría de naciones desarrolladas. Sus negocios no fueron tan exitosos como habrían esperado, destacando más su labor como "conseguidores" de concesiones y contratas que como administradores. Pese a ello, su nombre ha quedado ligado al del amanecer de la industria en Francia y en España.

En definitiva, la saga de los Manby ilustra a la perfección el espíritu de la cada vez más poderosa burguesía europea, que recurriendo a mecanismos de reproducción de la riqueza extraños a la aristocracia tradicional, consiguió hacerse un lugar en la economía, para con el tiempo alcanzar la relevancia social y el peso político que le correspondían. Ahora bien, conscientes de que no podían plantear su oposición como un choque frontal con la nobleza, se asociaron con ella, incorporándola a las juntas de gobierno y de administración de las sociedades que crearon, al mismo tiempo que intentaban imitar su estilo de vida. Les unía el estatus y el prestigio social, pero les separaban dos elementos fundamentales: el derecho de cuna, del que esta burguesía carecía, aunque ya no lo necesitaba; y sobre todo la conciencia de que solo la circulación de capital, la diversificación de las inversiones y la apuesta por los sectores aún por explotar ofrecían la verdadera oportunidad para consolidar su posición. 


\section{AGRADECIMIENTOS}

Los autores desean dar las gracias a los evaluadores externos del artículo, porque sus comentarios han permitido mejorar su contenido e incorporar aspectos esenciales de la biografía de los personajes estudiados. Esta investigación forma parte de los resultados del Proyecto I+D+I de Excelencia PID2020112844GB-100, "El gas en la Europa Latina: una perspectiva comparativa y global (1818-1945)", financiado por el Ministerio de Ciencia e Innovación del Gobierno de España y Fondos FEDER. Asimismo,

\section{NOTAS AL FINAL}

1 Solo sabemos que fue banquero en la Isla de Wight.

2 Recuperado de: https://www.oxforddnb.com/search?q=Manby\%2C+Aaron\&searchBtn=Search\&isQuickSearch=true [consultado el 14 de mayo de 2020].

3 Algunas fuentes sostienen que en realidad su participación en la siderurgia de Charenton se remonta a 1819 (Biographical Dictionary, ed. 2008, pp. 431-432).

4 Recuperado de: https://www.oxforddnb.com/search?q=Manby\%2C+Aaron\&searchBtn=Search\&isQuickSearch=true [consultado el 14 de mayo de 2020].

5 Bibliothèque Nationale de France (BNF), Journal de l'enregistrement et des domaines par Une Société d'Employés Supérieurs de l'Administration, n. 1536, 11 de junio de 1841; n. 1645, 22 de julio de 1844; La Gaceta Industrial, p. 184, 31 de mayo de 1869.

6 Staffordshire Record Office (SRO), D1288/B/8/9-30. "The Manby Family and the "Aaron Manby" [mostly produced for the centenary of Manby's iron steamship]. Informe para la reproducción del Aaron Manby con motivo del centenario de su construcción. 3 de marzo de 1922.

7 La llustración Española y Americana, n. XVI, año XVII, p. 262, 24 de julio de 1873.

8 El Genio de la Libertad, n. 169, p. 3, 14 de julio de 1856.; La Discusión, n. 3.101, p. 1, 3 de febrero de 1866.

9 Recuperado de: https://www.oxforddnb.com/ view/10.1093/ref:odnb/9780198614128.001.0001/odnb-9780198614128-e-17918 [consultado el 20 de mayo de 2020].

10 Recuperado de: https://www.oxforddnb.com/search?q=Manby\%2C+Aaron\&searchBtn=Search\&isQuickSearch=true [consultado el 14 de mayo de 2020].

11 London Metropolitan Archives (LMA), Imperial Continental Gas Association, "Copy letters in and out. Include to and from Sir William Congreve concerning the possiblity of lighting contracs in Cadiz, Spain; Rio de Janeiro, Brazil; and the Netherlands. 1824-1825". Cádiz, 13 de diciembre de 1824.

12 La Esperanza, n. 372, p. 4, 19 de diciembre de 1845; El Heraldo, n. 1.152, p. 4, 15 de marzo de 1846. En esta publicación se recoge el nombre de los integrantes de la Junta Directiva, presidida por el marqués de Salamanca e integrada por Edward O. Manby y William Partington Hurts, entre otros socios. de los resultados de la Red Temática "INGEURSUR: Ingenieros y modernización de la Europa del Sur (Siglos XVIII-XX)", financiada por el Vicerrectorado de Investigación y Transferencia de la Universidad de Málaga. Igualmente, es el resultado de la estancia de investigación realizada en 2017 por Mercedes Fernández Paradas en The Cañada Blanch Centre for Contemporary Spanish Studies de la London School of Economics and Political Science, dedicada al tema de investigación "La influencia de la industria de gas británica en España: capital humano y tecnología”.

13 Gaceta de Madrid, n. 4.307, p. 4, 30 de junio de 1846; El Tiempo, n. 649 (5), 2 de julio de 1846.

14 The National Archives (TNA), Bill, Interrogatories, "Denuncia de Deeley \& Thomas contra Edward O. Manby y William Partington Hurts, 4 de mayo de 1853.

15 Archivo de Protocolos Notariales de Sevilla (APPNNS), Escritura de venta de edificio de don Guillermo Partington a don Jaime Federico Gregory, 1852.

16 El Clamor Público, n. 795, p. 3, 19 de diciembre de 1846

17 Gaceta de Madrid, n. 4.524, p. 4, 2 de febrero de 1847; n. 4.742 , p. 4,8 de septiembre de 1847 ; n. 4743 , p. 4,9 de septiembre de 1847.

18 El Clamor Público, n. 1.591, p. 4, 11 de enero de 1849. La España, n. 673, p. 1, 19 de junio de 1850.

19 Archivo de la Oficina Española de Patentes y Marcas (AOEPM), PR446, Certificado de introducción de la fabricación de sustancias espirituosas aplicables al calentado, alumbrado y otros usos, Madrid, 23 de marzo de 1849.

20 AOEPM, PR448, Certificado de introducción de un procedimiento para la fabricación del pan por medio de hornos continuos aerotermos, Madrid, 2 de junio de 1849.

21 AOEPM, PR493, Certificado de introducción de un proceder para cocer ladrillos, tejas y otras materias por medio de hornos llamados de Ainslie, Madrid, 7 de marzo de 1850.

22 AOEPM, PR 815, Privilegio de invención para un nuevo procedimiento que permitirá separar los minerales de sus gangas, Madrid, 9 de noviembre de 1850.

23 AOPEM, PR 821, Privilegio de introducción para mejoras en aparatos y procederes de destilaciones de carbón de piedra y otras sustancias en la fabricación de gas para el alumbrado, calentado u otros usos, Madrid, 6 de enero de 1851.

24 El Heraldo, n. 2.333, p. 4, 28 de diciembre de 1849.

25 El Heraldo, n. 3.052, p. 2, 18 de abril de 1852; El Clamor Público, n. 1.858, p. 1, 12 de septiembre de 1858.

26 Diario de Sesiones de las Cortes. Congreso de los Diputados (DSCCD), n. 71, "Interpelación del Sr. Martín sobre los perjuicios que causa la interpretación de algunos artículos de la ley de minas. Discurso del señor Martín explanándola", pp. 1.081 y ss., 12 de enero de 1861.

27 La Iberia, n. 333, p. 2, 18 de julio de 1855.

28 Gaceta de los Caminos de Hierro, n. 5, año 1, p. 74, 15 de mayo de 1856.

29 El Clamor Público, n. 2.202, p. 2, 10 de septiembre de 1851.

30 La lberia, n. 313, p. 2, 25 de junio de 1855. 


\section{BIBLIOGRAFÍA}

Alayo Manubens, J.C. \& Barca Salom, F.X. (2017), "Las técnicas de fabricación utilizadas en las fábricas de gas españolas (1852-1972)". En: Bartolomé Rodríguez, I.; Fernández-Paradas, M.; Mirás Araujo, J. (eds.), Globalización, nacionalización y liberalización de la industria del gas en la Europa latina (siglos XIX-XXI), Madrid, Marcial Pons, pp. 141-171.

Arroyo, Mercedes (2000), "Tècnics i tecnología de gas a la Catalunya del segle XIX", Quaderns d'Història de l'enginyeria, IV, pp. 45-72.

Arroyo, Mercedes (2001), "Banca, infraestructuras urbanas y estrategias empresariales. La fábrica de gas de Málaga (1923-1940)". En: Actas del 3er Congreso de historia catalano-andaluza. Cataluña y Andalucía, 1898-1939, Barcelona, Ediciones Carena, pp. 297-325.

Biographical Dictionary of Civil Engineers in Great Britain and Ireland (ed. 2008), vol. 3, 1830-1890, London, M.M. Chrimes and Others, pp. 431-432.

Chaloner, W.H.; Henderson, W.O. (1995), "The Manbys and the Industrial Revolution in France 1819-84", History of Engineering and Technology, 30, pp. 63-75.

Chatwin, Amina (1972), "Early Years of the Cheltenham Gas Light and Coke Company and the Beginning of the Tewkesbury Gas Light Company", Gloucestershire Society for Industrial Archaelogy Journal, pp. 10-35.

Cotte, Michel (2010), "Le rôle des ovuriers et entrepreneurs britanniques dans la décollage industriel français des années 1820", Documents pour l'histoire des techniques, 19, pp. 119-130.

VV. AA., "Élements biographiques d'après Victor Chauvin" (1866), Histoire des lycées et collèges de Paris, Paris, Hachette, pp. 187-189.

Fàbregas, Pere A. (1993), Un científico catalán en el siglo XIX. José Roura y Estrada (1787-1860): enseñanza técnica y gas de alumbrado en la modernización del país, Barcelona, Enciclopèdia Catalana.

Fàbregas, Pere A. (2017), "La estrategia de la implantación de la industria del gas en España (1826-2010)". En: Bartolomé Rodríguez, I.; Fernández-Paradas, M.; Mirás Araujo, J. (eds.), Globalización, nacionalización y liberalización de la industria del gas en la Europa latina (siglos XIX-XXI), Madrid, Marcial Pons, pp. 21-44.

Fernández-Paradas, Mercedes (2009), “Empresas y servicio del alumbrado público por gas en España (1842-1935)”, Transportes, Servicios y Telecomunicaciones, 16, pp. 108-131.

Fernández-Paradas, Mercedes (2015), La industria del gas en Cádiz (1845-2012), Fundación Gas Natural Fenosa, LID Editorial.

Fernández-Paradas, Mercedes; Larrinaga Rodríguez, Carlos; Matés Barco, Juan Manuel (2021), "Los ingenieros y el suministro de gas en la España del siglo XIX", Revista de Historia Industrial (en prensa).

Fernández-Paradas, Mercedes; Pinto Tortosa, Antonio Jesús (2019), “Combining Entrepreneurship Spirit and Power Links. Melitón Martín and the Dawn of Spanish Gas Lighting". En:
Technology and Power. 46th Symposium of ICOHTEC, Katowice: Polonia, 22-27 de julio de 2019.

Fernández-Paradas, Mercedes; Pinto Tortosa, Antonio Jesús (2020), “¿Emprendedor o "conseguidor"? William Partington Hurts y el despegue de la industria española del gas". En: Bartolomé Rodríguez, I.; Fernández-Paradas, M.; Mirás Araujo, J. (eds.), Cercanas pero distintas. La desigual trayectoria de la industria del gas en las regiones del sur de Europa (siglos XIX-XX), Madrid, Marcial Pons, pp. 349-365.

García de la Fuente, D. (1990), “La Real Sociedad Económica de Cádiz y el gas de alumbrado”, Cuadernos de Historia, 20, pp. 1-15.

García de la Fuente, D. (1998), La historia del gas en Granada Del gas "Lebon" al gas natural, Sevilla, Gas Andalucía.

García de la Fuente, D. (2002), La luz de gas en Alcoy. Del farol de aceite al foco eléctrico, Alcoy, Fundación José y Ana Arroyo.

García Hourcade, J.L. (2010), “Melitón Martín, un famoso desconocido. Lección inaugural del curso académico 2009-2010", Estudios Segovianos. Boletín de la Real Academia de Historia y Arte de San Quirce Asociada al Instituto de España, LII (109), pp. 20-59.

Heredia-Flores, V.M. (2013), “Municipalización y modernización del servicio de abastecimiento de agua en España: el caso de Málaga (1860-1930)", Agua y Territorio, 1, pp. 103-117.

Lalvani, Kartar (2016), The Making of India. The Untold Story of British Enterprise, London - New York, Bloomsbury.

Lesseps, Ferdinand de (1856), Percement de l'isthme de Suez. Rapport et projet de la commission internationale, Paris, Chez Henri Plon Éditeur.

"Manby, Aaron", Oxford Dictionary of National Biography, ed. 2020. Recuperado de https://www.oxforddnb.com/search?$\mathrm{q}=$ Manby\%2C+Aaron\&searchBtn=Search\&isQuickSearch=true [consultado el 14 de mayo de 2020].

"Manby, Charles", Oxford Dictionary of National Biography ed. 2020. Recuperado de https://www.oxforddnb.com/ view/10.1093/ref:odnb/9780198614128.001.0001/odnb-9780198614128-e-17918 [consultado el 20 de mayo de 2020].

Manby, Edward Oliver; Partington Hurts, William (1846). Memoria sobre el Alumbrado de Gas de la Villa de Madrid, Madrid, Imprenta y Fundición de Don Eusebio Aguado, 1846.

VV. AA., "Obituary. Edward Oliver Manby, 1816-1864" (1865), Minutes of the Proceedings of the Institution of Civil Engineers, 24, pp. 533-534

VV. AA., “Obituary. Josep Lane Manby, 1814-1862” (1863), Minutes of the Proceedings of the Institution of Civil Engineers, 22, pp. 629-630

Payen, Jacques (1969), Capital et machine à vapeur au XVIIle siècle: les Frères Perier et l'introduction en France de la machine à vapeur de Watt, Histoire des Sciences et des Techniques, vol. I, Paris, Mouton.

Raveux, Olivier (1994), “El papel de los técnicos ingleses en la industria metalúrgica y mecánica del norte del Mediterráneo (1835-1875): una primera aproximación", Revista de Historia Industrial, 6, pp. 143-161. 
Robinson, Robb (2009), "The Cookman Story: reform in Hull and the United States", Far horizons - to the ends of the Earth, Hull, Maritime Historical Centre - University of Hull.

Simón Palmer, M. C. (1989), El Gas y los madrileños, Madrid, Gas Madrid.

Simón Palmer, M. C. (2011), La Real Fábrica de Gas de Madrid, Barcelona, LID Editorial.

Sudrià, C. (1983), "Notas sobre la implantación y el desarrollo de la industria del gas en España, 1840-1901", Revista de Historia Económica, 2, pp. 97-107.

The Repertory of Arts, Manufactures, and Agriculture (1818), London, Printed for J. Wyatt - The Repertory Office - Haton Garden, pp. 11-16.
Thomas, Russell (2014), Gasworks Profile A: The History and Operation of Gasworks (Manufactured Gas Plants) in Britain, Contaminated Land - Aplications in Real Environments (CLAIRE), London.

Torquemada Daza, J.A. (2006), “La Compañía de los Ferrocarriles Andaluces en la cuenca minera de Bélmez. Un análisis de su actividad minera, social, económica y política en el norte de la provincia de Córdoba". En: IV Congreso de Historia Ferroviaria, Málaga, Universidad de Málaga, pp. 1-26.

Tortella, Teresa (2000), Una guía de fuentes sobre inversiones extranjeras en España (1780-1914), Madrid, Banco de España. 